\title{
A Cloud-to-Edge Approach to Support Predictive Analytics in Robotics Industry
}

\author{
Simone Panicucci ${ }^{1}\left(\mathbb{D}\right.$, Nikolaos Nikolakis $^{2}{ }^{(0)}$, Tania Cerquitelli ${ }^{3, *}{ }^{-1}$, Francesco Ventura $^{3}{ }^{(0)}$, \\ Stefano Proto ${ }^{3}{ }^{-}$, Enrico Macii ${ }^{4}\left(\mathbb{D}\right.$, Sotiris Makris ${ }^{2}$, David Bowden ${ }^{5}$, Paul Becker ${ }^{6}$, \\ Niamh $\mathrm{O}^{\prime}$ Mahony ${ }^{3}$, Lucrezia Morabito ${ }^{1}$, Chiara Napione ${ }^{1}$, Angelo Marguglio ${ }^{7}{ }^{\circ}$, \\ Guido Coppo ${ }^{8}$ and Salvatore Andolina ${ }^{8}$ \\ 1 COMAU S.p.A., 10095 Turin, Italy; simone.panicucci@comau.com (S.P.); \\ lucrezia.morabito@comau.com (L.M.); chiara.napione@comau.com (C.N.) \\ 2 Laboratory for Manufacturing Systems \& Automation, Department of Mechanical Engineering \\ and Aeronautics, University of Patras, 26504 Patras, Greece; nikolakis@lms.mech.upatras.gr (N.N.); \\ makris@lms.mech.upatras.gr (S.M.) \\ 3 Department of Control and Computer engineering, Politecnico di Torino, 10129 Turin, Italy; \\ francesco.ventura@polito.it (F.V.); stefano.proto@polito.it (S.P.); Niamh.Omahony@dell.com (N.O.) \\ 4 Interuniversity Department of Regional and Urban Studies and Planning, Politecnico di Torino, \\ 10125 Turin, Italy; enrico.macii@polito.it \\ 5 DELL EMC, P31 D253 Cork, Ireland; david.bowden@dell.com \\ 6 Fraunhofer Gesellschaft zur Förderung der angewandten Forschung, 52074 Aachen, Germany; \\ paul.becker89@gmail.com \\ 7 Engineering Ingegneria Informatica S.p.A., 90146 Palermo, Italy; Angelo.Marguglio@eng.it \\ 8 SynArea Consultants S.r.l., 10153 Turin, Italy; guido.coppo@synarea.com (G.C.); \\ salvatore.andolina@synarea.com (S.A.) \\ * Correspondence: tania.cerquitelli@polito.it
}

Received: 24 December 2019; Accepted: 12 March 2020; Published: 16 March 2020

\begin{abstract}
Data management and processing to enable predictive analytics in cyber physical systems holds the promise of creating insight over underlying processes, discovering anomalous behaviours and predicting imminent failures threatening a normal and smooth production process. In this context, proactive strategies can be adopted, as enabled by predictive analytics. Predictive analytics in turn can make a shift in traditional maintenance approaches to more effective optimising their cost and transforming maintenance from a necessary evil to a strategic business factor. Empowered by the aforementioned points, this paper discusses a novel methodology for remaining useful life (RUL) estimation enabling predictive maintenance of industrial equipment using partial knowledge over its degradation function and the parameters that are affecting it. Moreover, the design and prototype implementation of a plug-n-play end-to-end cloud architecture, supporting predictive maintenance of industrial equipment is presented integrating the aforementioned concept as a service. This is achieved by integrating edge gateways, data stores at both the edge and the cloud, and various applications, such as predictive analytics, visualization and scheduling, integrated as services in the cloud system. The proposed approach has been implemented into a prototype and tested in an industrial use case related to the maintenance of a robotic arm. Obtained results show the effectiveness and the efficiency of the proposed methodology in supporting predictive analytics in the era of Industry 4.0.
\end{abstract}

Keywords: machine learning; predictive maintenance; visualization techniques; data management; big data architecture 


\section{Introduction}

The industrial world is undergoing a shift to industry 4.0, where physical processes merge with their virtual counterparts towards increased flexibility and predictability. In this context, data acquisition and processing can enable smart functionalities. Data analysis techniques, such as clustering and anomaly detection, are used for enabling predictive analytics. Hence, powerful and reliable cyber-physical system (CPS) architectures [1] are becoming prominent to effectively analyze such large amounts of data, creating insight into the production process, and, thus, enabling its improvement, as well as competitive business advantages.

The proposed architecture, the "SERENA" system, can identify the symptoms of imminent machine failure, through the characterization of the current dynamics of the process/machine using data collected from the factory. A scalable and modular approach has been taken in the design of the architecture, decoupling the overall design from any specific set of technologies. In particular, there have been designed and implemented three different services for enabling predictive analytics, including a self-assessment service to automatically detect the drift in the data under analysis and trigger the building of a new predictive model, and a data-driven estimation of the remaining useful life.

This prototype has been validated in a real-world scenario involving anomaly detection on a robotic axis and concerning the maintenance requirements caused by wrong belt tensioning effect. The visualization service enables a real-time data stream and machine visualization, while the predictive analytics services generate the estimated remaining useful life (RUL) value, which is consumed by the scheduling service to proactively schedule the maintenance activities.

This paper is organized as follows. Section 2 discusses the state-of-the-art architectures and methodologies in the era of Industry 4.0. Section 3 describes the proposed architecture enabling predictive maintenance in industrial environments equipped with Internet of Thing devices. In addition, Section 4 presents the services of the SERENA system, while Section 5 introduces the industrial use case. Section 6 presents some results achieved by exploiting the proposed architecture in a robotic industry. Finally, Section 7 draws conclusions and discusses future developments of the proposed approach.

\section{Related Work}

The expression Industry 4.0 has been minted by research and industrial partners during the last decade. In this context, a large variety of studies and research projects have been conducted. The work in [2] shows an exemplary case study on big data analytics in industrial production environments. The outcome of this is a cross-industry methodology, capable of process improvements with data mining. In [1] a distributed system architecture was used to enable predictive maintenance on a self-tuning engine by combining dynamic use of different prediction algorithms with data interpretation functionalities to allow better understanding by the end user. While in [3] an overview of IoT platforms with applications in enhanced living environments and in healthcare systems is given highlighting the applications, the challenges, the opportunities of these new technologies.

The differences between multi-class classifiers and deep learning techniques are discussed in [4]. A comparative experimental analysis of exploratory techniques was presented in [5]. The scalability of big data methodologies is discussed in [6] using an example from the energy domain industry. The authors of [7] present a proposal for on-demand remote sensing of data to save network and processing capacities by extracting features as soon as possible in the data flow.

The linking between existing production system, their legacy systems and advanced Internet of Things (IoT) technologies is discussed in [6]. For this purpose, virtualization and a cloud-based micro-service architecture are key technologies. The topic of distributed micro-service architectures for big data applications is also discussed in [8]. Therein, the author describes an approach done by the Open Geospatial Consortium (OGC) to process large quantities of data collected by earth observation satellites. By bringing micro-services to the place where data is captured and stored 
instead of moving these large quantities of data to one central processing node, unnecessary data transport can be significantly reduced. In [9] a scalable full-stack distributed engine tailored to energy data collection and forecasting in the smart-city context enabling the integration of the heterogeneous data measurements gathered from real-world systems and the forecasting of average power demand. Moreover, related to fog computing, in [10] the authors introduce a new architecture enabling on-demand computation to offload the computation in the cloud architecture, reducing unnecessary network overhead, by properly selecting the most effective edge devices as computation delegates.

In the context of maintenance strategies, the condition based approach is considered as effective [11], but also complex to implement and integrate to a production system [12]. However, the determination of the current equipment condition is an important issue in the maintenance process chain. Using a combination of raw data and intelligent trend filtering, anomalies can be detected in various ways, as discussed in [13]. Usually identified anomalies or the status of machine's degradation are expressed in terms of a machine's remaining useful life (RUL). In that way, a complex statistical analysis is directly mapped to a value with physical meaning for the maintenance personnel, responsible for further actions. The authors of $[14,15]$ explore ways to estimate the RUL and derive a detailed maintenance scheduling strategy. It is important to note that to adequately estimate an equipment's condition using raw data as a basis, a dataset of proper quality is necessary. This requires data corresponding to both normal and abnormal working conditions. Methods for data quality improvements are discussed by the authors of [16]. The authors describe how datasets can be visualized, grouped, classified, clustered and evaluated in order to detect and remove outliers.

This paper extends the work presented in [17] by (i) presenting an up-to-date architecture of the SERENA system, (ii) describing in further detail the main components of the predictive analytics service, and (iii) providing additional experimental results.

\section{Proposed Architecture}

The SERENA system is built on a lightweight and flexible micro-services architecture as shown in Figure 1, with the various applications implemented as independent Docker services. The services are integrated to seamlessly support maintenance engineers and personnel by providing the following main functionalities: (i) predictive maintenance analytics; (ii) dynamic maintenance scheduling; and (iii) assisted plant maintenance, using augmented reality devices. Communications are facilitated through a central message broker. With respect to the cloud-based nature of the architecture and its services, the SERENA data model is based on a JSON-LD [18] mapping of the MIMOSA XML schema [19]. A Docker orchestration layer manages the instantiation and life cycle of the services. The adoption of virtualization technologies like Docker, creates an agnostic middle-ware, enabling the SERENA system to be deployable on a wide variety of host infrastructures while providing scalability and resiliency. The Docker orchestration layer also provides the overlay network that allows the services to transparently communicate, irrespective of where they are deployed.

A standard practice for companies not providing IT products or services, is renting cloud space and web servers to avoid the related capital and maintenance cost, and it is obvious that production equipment and sensors are at a distance from the actual rented cloud infrastructure. With respect to the aforementioned point, the SERENA architecture extends the IoT cloud concept to directly include these devices. This is accomplished by wrapping the edge applications as Docker services, and managing their deployment and lifecycle from the Docker swarm as docker workers or managers in case of a complex swarm(s). This in turn can enable workload balancing in terms of deploying and executing docker applications to the appropriate network nodes, as well as managing the activation or deactivation of additional services at a certain node. In case of network bandwidth or latency constraints, the service can be moved to the edge of the cloud to analyze and convert the raw data into features, at source; alternatively, if high performance compute resources are required, such as tensor processing units (TPUs) for training machine learning (ML) models, services can be run on the central cloud infrastructure or in remote public clouds. Thus, the SERENA architecture considers both cloud 
and edge nodes, all being part of the same hybrid SERENA cloud system, centrally and holistically managed as one or more Docker swarm service network.

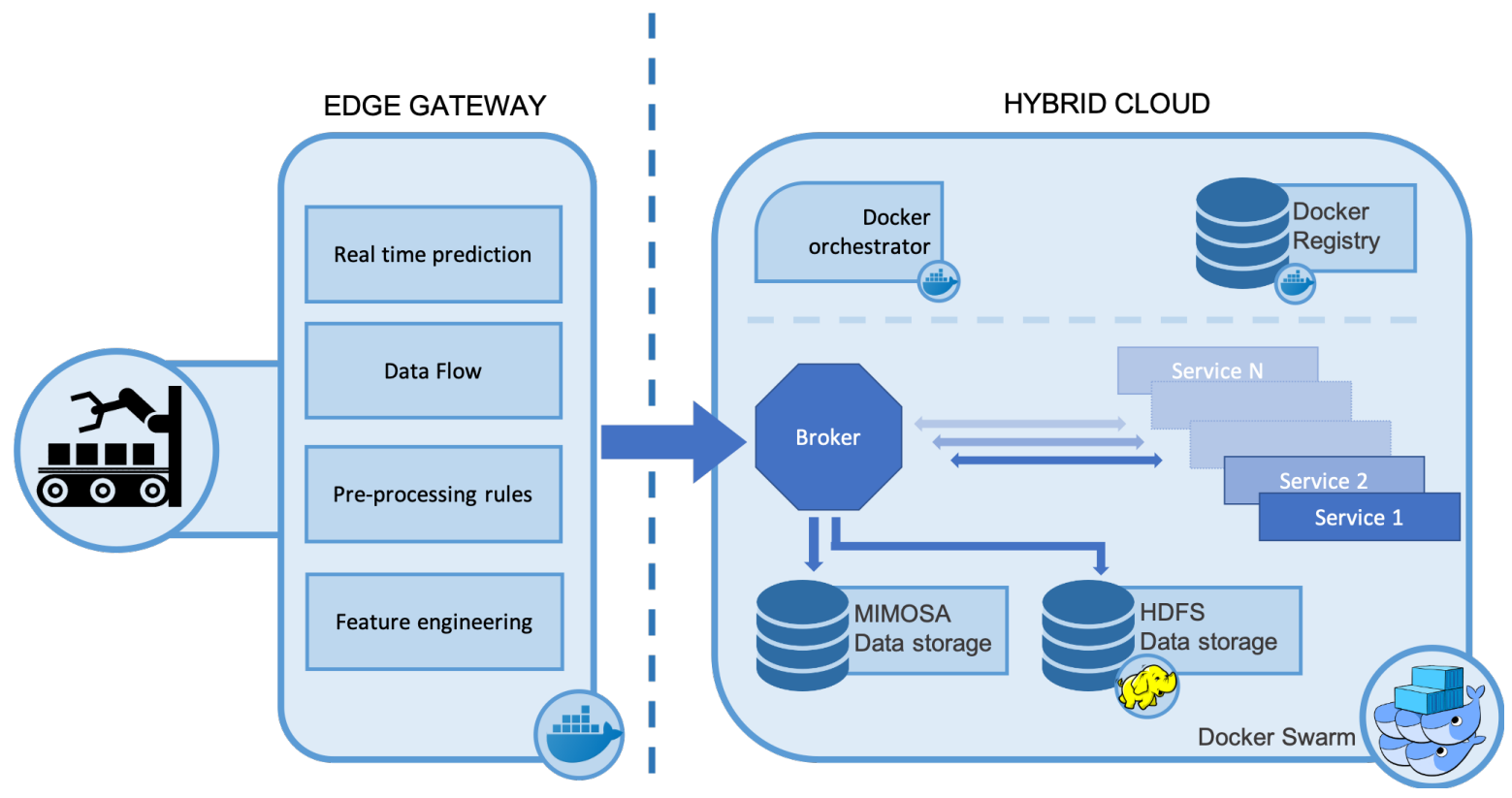

Figure 1. The SERENA Architecture.

In the context of the SERENA project, a specific gateway has been designed and developed based on an Intel NUC, which has 8 GB of RAM and an Intel i5, as well as some analog inputs. The edge component, referring to an edge gateway (hardware and software), allows building real-time streaming applications, reliably collecting data among different heterogeneous systems or applications and transforming or reacting to the streams of data collected in the plant. It can scale in both data size and velocity. The edge gateway is located on the factory floor, collecting sensor data coming from industrial equipment as well as context information and the operating condition of the equipment. It includes the following key functionalities:

- The data flow engine, managing the data streams;

- A feature engineering component pre-processing incoming raw data into values that are of greater meaning for predictive analytics, such as the current average $/ \mathrm{rms}$ value instead of simple amplitude;

- The rules for performing the aforementioned pre-processing;

- A pre-trained predictive analytics model providing real time predictions/classifications.

All components are deployed as docker containers under the control of a docker orchestration manager. Wrapping edge functionalities as docker services give the system the flexibility to execute the application workloads wherever it makes the most sense: If there are network bandwidth or latency constraints, the service can be moved to the edge of the cloud to analyze and convert the raw data into feature engineering, at source; alternatively, if high-performance computing resources are required, such as tensor processing units (TPUs) for training machine learning (ML) models, services can be run on the central cloud infrastructure or in remote public clouds.

The SERENA system has been designed and implemented aiming to be technology-independent. The reason for this is to allow any integrator or developer to customize it and tailor its reference technologies depending on case-specific technology limitations. Whilst a reference architecture and implementation are provided, the system has been designed in such a way as to give the developer the freedom to choose the technologies that conform to their own corporate guidelines or meet specific implementation challenges. 
In particular, the technologies used for implementing the SERENA system during the proof of concept stage along with the data storage mechanisms are transparent to the other services. Thus, older technologies can be swapped for newer more powerful ones, without having to rebuild the entire system. As an example, the preferred data storage platform is HDFS [20], but other databases, such as Cassandra [21], may be preferred in specific cases.

\section{Services}

The architecture presented in the previous section has been designed to enable predictive analytics of industrial equipment. Towards that, a set of application services has been integrated. In the following sections the main services are presented in greater detail.

\subsection{The Predictive Analytic Service}

A general-purpose service suited to fulfill the condition monitoring and maintenance needs of modern companies in the context of industry 4.0 is proposed. The architecture of the analytical service proposed in this research, reported in Figure 2, is flexible and it can be customized for any other use cases of interest (e.g., [22]). The service is based on four main steps: feature engineering, predictive analytics, model validation, self-assessment.

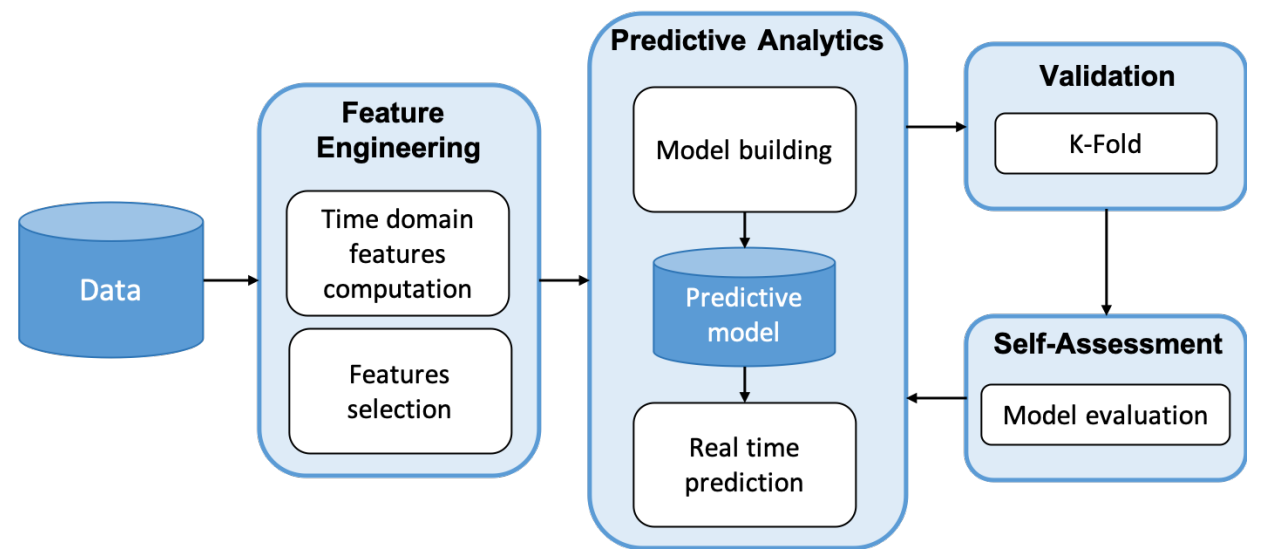

Figure 2. Analytic architecture.

Feature engineering. Industrial sensors monitor many production processes, that are usually repeated periodically and characterized by a specific duration. The feature engineering component is in charge of transforming and processing raw sensor data to extract the main features describing the signals. They are identified at the first stages of the raw data analysis process (i.e., clustering, correlation analysis, PCA, other) and implemented at a latter stage to reduce the amount of data communicated from the edge to the cloud. Specifically, each monitored cycle is divided in several segments over the time domain, to better extract the variability separately for each sub-cycle. Each segment is thus characterized by many statistical features (e.g., mean, standard deviation, quartiles, kurtosis, skewness, root mean squared error, sum of absolute values, number of elements over the mean, absolute energy, mean absolute change). These features are then used by the other steps of the analytical process to predict the outcome for each cycle.

Since the great number of computed statistical features, it is possible that the time domain feature computation step produces a huge number of attributes and that, in some cases, this can affect the performance of the successive analysis. However, since it is possible that some features are highly correlated with some others in the dataset, the information gained by these attributes could be redundant and it can possibly produce noise in the model building phase. Thus, to select the features that contain the most valuable information, the analytics engine includes a feature selection technique based on the Pearson correlation test [23]. Computing the correlation of each couple of attributes and removing those that are correlated the most, on average over all the (other) attributes, 
it is possible to identify which are the attributes that can be discarded without losing any accuracy in the model construction.

Predictive analytics. The predictive analytics block has the role to learn from the historical dataset and build a prediction model, to forecast the correct label for new incoming sensor data in the real time prediction phase. The model building process exploits a training phase necessary to extract the latent relations which exist between an historical set of signals and its labels (events to predict). The information contained in the features is analyzed by different scalable and transparent classification algorithms, available in the Apache Spark framework [24,25]: (i) Decision Tree [26] (DT), (ii) Random Forest [27] (RF), (iii) Gradient Boosted Tree [28] (GBT). The chosen classification algorithms have been specifically picked for their interpretability: domain experts can indeed easily understand and inspect the main relationships between the input data and the outcome of the predictions. Once the model is built, the labels (and the probability to belong to a specific label) for new incoming sensors data can be forecasted by the real time prediction. The trained model is pushed down to the edge to fulfill the need for short response time of the predictive models, in order to enable in-time corrective/preventive actions to take place on the shopfloor. Also for safety reasons for the human personnel as well as for preventing any robot/machine damage, that would be associated to additional expenditures. This would need a pre-trained model deployed close to the robot, getting data and processing in short time, providing results easy to understand and interpret by the human(s) in close proximity outputs.

Model validation. The performance of the predictive model built in the previous step has to be evaluated by the Validation block. In such phase, all the trained classification models are validated using the Stratified K-Fold Cross validation strategy and the values of precision, recall and f-measure are calculated for each class of interest. This step is necessary to understand which algorithm better fits the requirements of the given classification task (i.e., have higher values for the f-measure and the other metrics). The indexes computed to evaluate the training of the model require the information of the real class of the test data, namely a subset of labeled data kept apart during the training phase through a certain sampling strategy. Because of this reason, these approaches are not eligible to be used to evaluate the model performances on unlabeled data over time.

Self-assessment. It is common, especially in industrial production environments, that the nature of the collected data changes over time due to equipment degradation, the adoption of new machinery or to some environmental factors. The Self-assessment phase allows to identify if, due to changes in the production environment or in case of new labels not included in the dataset, the predictive model performance has degraded and so decides whether to trigger the update and retrain of model with newly arrived data.

The main idea behind the self-assessment approach is to exploit the changes in the geometrical distribution of the data over time to identify when the predictive model is no more performing as expected. Moreover, in real use cases, the ground truth label is often not available, thus, only unsupervised techniques can be exploited. The proposed methodology exploits a new, scalable version of a well known unsupervised index able to measure the intra-cohesion and inter-separation of clusters of data along. The exploited metric is the Descriptor Silhouette (DS) index proposed in [29]. It provides an approximated estimation of the Silhouette index. The DS index ranges in $[-1,1]$, as the standard Silhouette index, where a -1 represents a point that is distant from the distribution of the points to which it is assigned (bad assignment), while 1 describe a well assignment for the point in exam. Each record in the dataset is characterized by a DS score each time the self-assessment is executed, producing a Silhouette curve. Given a dataset characterized by class labels, it is possible to measure the cohesion and the separation of the data, before and after the collection of new incoming data. It has been proved experimentally that a variation of the geometric distribution of the incoming data w.r.t. the historical data corresponds to the presence of new unknown data. Then, the self-assessment service produces a measure of the percentage of degradation of the predictive model performance given by the following relation: 


$$
\operatorname{DEG}(c, t)=\alpha \times \operatorname{MAAPE}\left(\operatorname{Sil}_{t_{0}}, \operatorname{Sil}_{t}\right) \times \frac{N_{c}}{N}
$$

The degradation is calculated for each class $c$ learned by the predictive model and at specific timestamp t. The degradation is obtained calculating the Mean Arctangent Absolute Percentage Error (MAAPE) [30] between the DS curve calculated with the training data and the DS curve calculated with all the data collected until time $t$. This error is then weighted by the number of points predicted to belong to class $c\left(N_{c}\right)$ w.r.t. the cardinality of the whole dataset $(N)$. The coefficient $\alpha$ instead, give the sign to the degradation, describing if the mean silhouette is increased (negative sign) or decreased (positive sign), after the arrival of new unknown data. A positive sign of $\alpha$ represents a lower cohesion in the data, since the mean silhouette after the arrival of new data is lowered and thus the degradation is increased (the degradation is positive).

Remaining Useful Life estimation. A widely used indicator in industry about the condition of its assets is the Remaining Useful Life (RUL). The RUL of an asset can be defined as the time left to the end of its expected operational life and as it regards the functionality for which it was purchased for. The same asset operating under different conditions can be characterised by different degradation level, and as a result varying RUL values at the same point of time. As a consequence, the creation of a generic model, statistical or otherwise, for estimating the RUL value is extremely challenging if at all feasible [31]. Nevertheless, the accurate estimation of the RUL value is a key component towards enabling predictive maintenance of a system. Thus, several efforts are underway to provide a solution to this problem. However, state-of-the-art algorithms, such as liner regression models, Hidden Markov Models, or Long Short-Term Memory Neural Networks, require extensive and complex modelling and training to estimate the RUL value, with limited efficiency.

To that end and towards overcoming the aforementioned limitations, this work proposes a novel methodology for RUL estimation. In particular, data collected out of different phases of an asset operational life are used to model its behavior at specified and labelled timestamps and support the identification of deviations from its nominal or ideal operational profile and other as well labelled profiles. More specifically, data acquired at the deployment of an asset on a shop floor can be considered as an ideal operational profile from which degradation starts to gradually deteriorate its operational behavior. Afterwards and during its usage for production purposes, data are used to estimate the deviation from this nominal profile. The distribution of the nominal set of key variables is calculated. Then the probability of each selected variable to belong to the nominal distribution is estimate using the Gaussian kernel density estimation [32]. This probability representing the deviation of the two profiles can then be associated to the RUL indicator or similar, facilitating its estimation. The lower is the probability of each selected variable to belong to the data distribution of the correct functioning of the machinery, the faster is the degradation of object functionality, thus the lower is the RUL.

It should be mentioned that the set of selected as important variables for the aforementioned analysis can either be a-priori known, based on existing engineering/production knowledge or identified during the course of the data analysis by extracting features that may be used for pattern recognition and anomaly detection enabling in turn predictive maintenance activities. Afterwards, as the degradation level of the monitored equipment increases a drift from the initial distribution is detected, that can be associated to a decrease in the value of the RUL indicator. The deviation from the nominal profile or "distribution drift" is evaluated through the self-assessment service and for the selected features.

Hence, the estimation of the RUL value in the context of SERENA and as presented in this work relies upon two main factors: (1) the joint probability of the selected features with respect to their nominal profile or distribution, and (2) the presence of probability drifting in the collected data over 
time. Both factors consist functions of time $t$. At a random time $t$, the percentage of the RUL value can be defined as follows:

$$
R U L(t)=\frac{1}{N_{t}} \sum_{x}^{X(t)}\left((100-D E G(t)) \prod_{s}^{S} P\left(x_{s} \in K\left(X_{s}\left(t_{0}\right)\right)\right)\right.
$$

where

- $X\left(t_{0}\right)$ refers to the set of historical data collected from the the beginning of the operational life to a time $t_{0}$ corresponding to the nominal or ideal operational profile of the asset under consideration,

- $X(t)$ refers to the new data collected from $t_{0}$ to time $t$,

- $N_{t}$ corresponds to the number of new signals collected up to time $t$,

- $S$ is the set of relevant features for characterizing the degradation level of the asset,

- $K\left(X_{s}\left(t_{0}\right)\right)$ denotes the distribution of the feature $s \in S$ estimated from the historical data $X\left(t_{0}\right)$,

- $\quad P\left(x_{s} \in K\left(X_{s}\left(t_{0}\right)\right)\right.$ refers to the probability of feature $s$ as estimated out of a new set of data $x$ to belong in the distribution $K\left(X_{S}\left(t_{0}\right)\right)$,

- $D E G(t)$ is the overall degradation estimated by the data collected from $t_{0}$ to $t$ as measured out of the Equation (1).

As a result, Equation (2) estimates the percentage of RUL as a function of time by calculating the mean of the joint probabilities of each selected feature to belong to the ideal or nominal operational distribution, for each incoming set of data, weighted by 100 minus the percentage reflecting the probability drift at a time $t$. The contribution of the drifting probability is calculated as 100 minus the percentage of degradation, reflected in the collected data, since an opposite analogy is considered between the RUL and the drift values.

Using the aforementioned concept as a basis, the SERENA system is able to estimate the RUL value of an asset, machine, robot, other. Moreover, considering a human-centered cyber physical production system, the proposed approach supports its customised tuning and configuration by the human user towards addressing different scenarios and assets. It should be noted that an important aspect of the presented approach and as part of the implemented SERENA system, is the knowledge of the domain expert for configuring the system and identifying the relevant or important features for the analysis. This knowledge is considered as part of the experience acquired during production and not knowledge that an equipment manufacturer could provide, unless monitoring the asset during its operational lifetime.

In addition the proposed RUL estimation approach supports the correlation to the drift measured by Equation (1) to unknown or unmodelled knowledge collected over time through the analyzed features. This phenomenon is considered to represent degradation that cannot be effectively modelled due to its complexity and unknown correlation to the known asset variables and monitored characteristics.

\subsection{The Scheduling Service}

The result of the aforementioned predictive analytics service, a forecasted failure time horizon, is consumed by a scheduling service [33]. The aim of the service is to prevent the predicted failure, by assigning the required maintenance activities to operators within the given time-frame. This service can be extended to consider the current production plan, hence fitting the maintenance activities within a given time slot to optimize production outputs.

The scheduling service has been implemented in Java following a client-server model. The service inputs include the monitored equipment, RUL value, maintenance tasks, including precedence relations and default duration per operation experience, and a number of potential operators with their characteristics, such as experience level. The server side includes a multi-criterion decision making framework, evaluating the alternative scheduling configurations, ranking them and selecting 
the highest ranked one. The client side communicates with the server side via restful APIs, supporting editing of tasks, resources, equipment, time series visualization, and process plan Gantt visualization.

The process time required to create a new schedule depends on the complexity of the schedule, referring to the number of tasks, resources, and their dependencies, along with the evaluated criteria.

\subsection{The Visualization Service}

The aim of the visualization service is to present the data, coming from the manufacturing process, in an effective and intuitive manner. It is an essential support for the maintenance engineers, giving them the opportunity to evaluate the status of the remote manufacturing process, and to the maintenance operator, who is responsible for performing the maintenance activity.

The visualization service can be considered as a set of information pages that integrate data and plant information coming from the manufacturing process, with the results of the predictive analysis. To effectively present real-time errors and predictions to the user in an intuitive way, the service uses a simplified, but realistic, 3D representation of the machinery. In addition, the user can display maintenance guidance and information pertaining to the mechanical and electrical components of the machine.

A Unity application that provides a real-time animation of the RobotBox 3D model via a web browser has been designed and developed. The live animation of the 3D model is achieved through a real-time data feed from the RobotBox controller, and the predictive information and machine status is obtained from the other SERENA services. The status of the component of interest is highlighted on the 3D model, along with its RUL. The 3D Virtual Procedure is an animated step-by-step guide, which shows the correct procedure to perform the maintenance operation. The concept is to evolve the typical text-based maintenance manual into an interactive 3D maintenance visualization, and so collaboratively guide the operator through the maintenance procedure.

The visualization service is also charged to show to the end user the information produced by the analytic services. The visualization service and the analytic services communicate through RESTFUL APIs, enabling also the monitoring of the performance for the predictive models used in the production environment. The monitoring of the predictive models performance, as explained in previous sections, is an important activity that should be always taken into account when dealing with real applications. The visualization service enables access to the information related to this task simplifying the understanding by non-expert users and the reliability of the models in production environments.

The information about the predictive model performance are showed in an interactive dashboard. The dashboard reports the precision and the recall information for the predictive model in production. Moreover, the dashboard shows an interactive section that exploits bar charts to describe the degradation of the model performance over time, for each learned class. The user can interact with the dashboard to see the details about the degradation at a specific timestamp. For the selected timestamp and for each learned class, the dashboard shows the silhouette lines produced by the self-assessment service though an interpolated scatter plot. More dashboard details are reported in Section 6-Self-assessment.

\subsection{Cloud Management and Communication Services}

The cloud management and communication services provide the base functionality that implements the SERENA system.

Docker orchestrator service. The orchestrator controls the deployment and manages the life cycle of all services in the SERENA cloud. It ensures that the services are running and will redeploy them if part of the underlying infrastructure fails. Critical services, such as the central message broker, are deployed as resilient and scalable service clusters. The SERENA system implements its own local image registry, where the service images are stored. Running a local image registry reduces the security 
risk of directly accessing an Internet Docker registry, and ensures that the required images are always available from a trusted source.

As the edge gateways are part of the SERENA cloud, the orchestrator can automatically deploy services all the way to the edge. The analytics models are periodically retrained in the cloud, then wrapped as a Docker service and deployed to the gateways. The specific manufacturing process that the gateway supports is configured via the orchestrator using function description labels. Based on the labels the orchestrator will automatically deploy the correct services to the gateway.

Edge gateway services. The edge gateways collect and analyze raw sensor data from the manufacturing process, and are fully integrated parts of the SERENA cloud. Typically, two types of service are deployed to the gateway: a data flow engine and a pre-trained data analytics or predictive analytics. The data flow engine, based on Node-RED [34], collects raw sensor data from the manufacturing process and passes it to the analytics service. The analytics service converts the raw time series data into features and encoding them into JSON-LD. JSON-LD is an extension of JSON that supports Linked Data, which allows data and metadata to be combined into a single context. The data flow engine then forwards the JSON-LD to the central message broker for distribution to the other SERENA services.

Regarding predictive analytics, the gateway represents a core component since it allows to fulfill different use case requirements and/or constraints. In the case of low latency, prediction is not needed (as is required in case of mechanical reaction in the actual process) or analytics algorithms require a huge amount of historical data which does not fit in the gateway storage; we can both train and execute predictive algorithms in the cloud in order to better optimize hardware resources and not overload equipment close to production. Otherwise, if the application requires to have the results of analytics services with high-performance constraints, the SERENA architecture offers a mechanism to deploy to the local gateway a model, trained in the cloud, whose output could be used in near real-time applications to easily perform the prediction.

Central message broker service. The central message broker service is the main communication hub of the SERENA system. To ensure its scalability and high availability, it is deployed as a resilient Docker cluster service. The broker exposes REST, MQTT and web service endpoints, which distribute messages between the cloud services and the edge gateways. In addition, the broker is intended to act as the access point for external facilities. Security is an important feature of the SERENA system, and the broker, as the communications hub, provides secure channels between the gateways and cloud services. It also validates the authenticity of incoming messages, and whether the requester is authorized to use the requested service.

Repository services. The SERENA architecture provides a number of repositories which hold various types of data used by the system, including the features and raw data repositories as well as maintenance manuals, data artefacts and information required by other SERENA services, such as maintenance tasks for the scheduler. The raw data repository is used for training the predictive analytics models. In addition, a metadata repository is included for storing contextual information connecting distributed stored and collected data. The repositories are implemented as stateless Docker services, the state being stored in external virtual volumes. Implementing the repositories as stateless services, enables a plug-n-play and dynamic deployment like the other services in the system.

\section{Use Case Description}

Given the high throughput of automation lines and the great number of robots involved in production, a common issue is the robot belts, working continuously for days at high cycles per minute ratios, suffering tensioning problems. The belts are used as transmission for the robot axes, and setting the tension is crucial for obtaining high levels of performance and precision.

In factory plants, preventive maintenance policies are used to avoid production stops. Hence, the acquisition and analysis of failure data for predictive analytics is a challenge. To overcome the lack of failure data, since it would have been too complicated and expensive to manipulate an 
entire robot, a test-bed (Figure 3) has been created, the RobotBox. The RobotBox is made of a motor equipped with an encoder (to read the actual motor axis position), a belt, a gearbox reducer, and a $5 \mathrm{~kg}$ weight to simulate an actual robot application. All components have been taken from a six axis industrial robot.

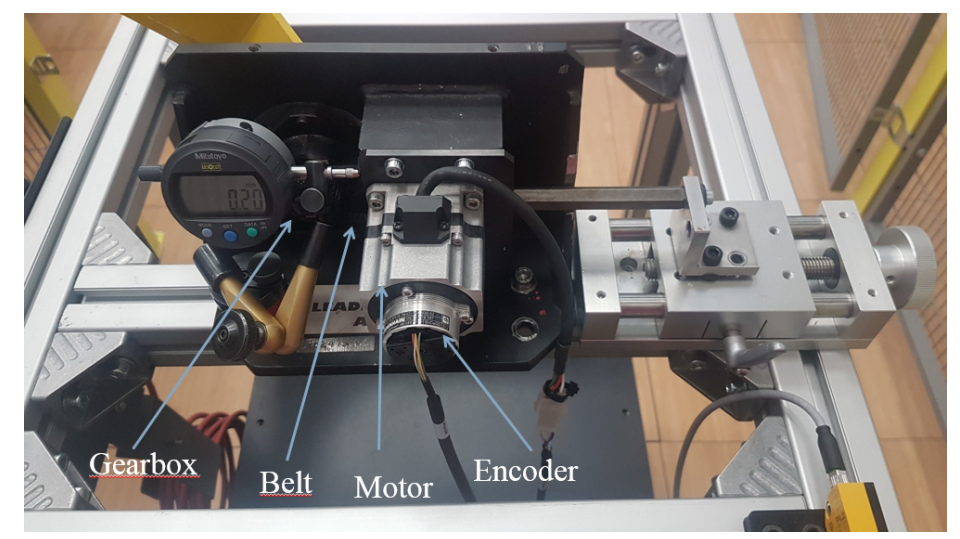

Figure 3. RobotBox components.

The decision to build the test-bed (RobotBox) instead of using an entire robot is not only due to the cost. Indeed, this simplification allows to better isolate the system from environmental factors such as weight of the links, inertia, vibration and so on. Thus, using the RobotBox allows to collect less noisy data and generalize the knowledge acquired to the entire robot axes.

In order to create a high quality dataset, changing the belt tension levels in a reproducible manner was necessary. Hence a slider was installed allowing a continuous change of the tension and a centesimal indicator, to measure the belt tensioning with respect to a pre-configured position. The distance between the gearbox and the motor is proportional to the belt tensioning.

In this work, only the current (in Amperes) used by the RobotBox motor is taken into account and used as input for the analytic component. The current is tracked by the RobotBox controller every 2 ms. Nevertheless, future works will consider fusing data of different sources.

The gateway collects data from the RobotBox and pre-processes the data in order to extract feature engineering for the analytics. The results of this processing step enriched by the result of already-deployed analytics models, remaining useful life or otherwise, is transmitted afterwards to the cloud node as a JSON file. Currently, and for the use case presented in this work, almost 1 GB of data are collected and transmitted per day, including raw data, extracted features, and analytics results of models already deployed at the gateway. Nevertheless, depending on the application, it could be sufficient to collect a smaller dataset with a greater time window.

Regarding computational power and bandwidth, the most impacting process is the prediction of the status of the machine, which is performed in runtime and at the gateway level. The response time, and hence the hardware setup requirements in terms of connectivity, data transmission, and processing/storage power, for the use case under discussion is expected to be within minutes. This is addressed by the aforementioned setup. More complex and time- or resource-consuming analyses, including the training and testing of new predictive models with new datasets as collected through time, take place at the cloud, and when a new model is created, this replaces the one deployed at the gateway.

\section{Experimental Setting and Results}

In this section the experimental setting and results related to the predictive analytics service are discussed in order to demonstrate its ability in correctly performing the prediction on real data. The analytics service, including both the model building phase and the real-time prediction, has been developed on the top of the big-data analytic framework Apache Spark [24], along with the scalable 
machine learning library MLLib [25]. The effectiveness of the proposed methodology is validated through a set of experiments, all performed on a Intel i7 8-core processor workstation, with a $32 \mathrm{~GB}$ main memory.

Using the slider installed on the RobotBox, 5 different classes of tensioning have been defined: classes 0 and 1 refer to low tensioning, class 2 to correct tensioning and classes 3 and 4 to high tensioning. Figure 4 shows an example of the current amplitudes values in Amperes for different level of tensioning. Table 1 shows the number of cycles in the dataset under analysis, divided by class.

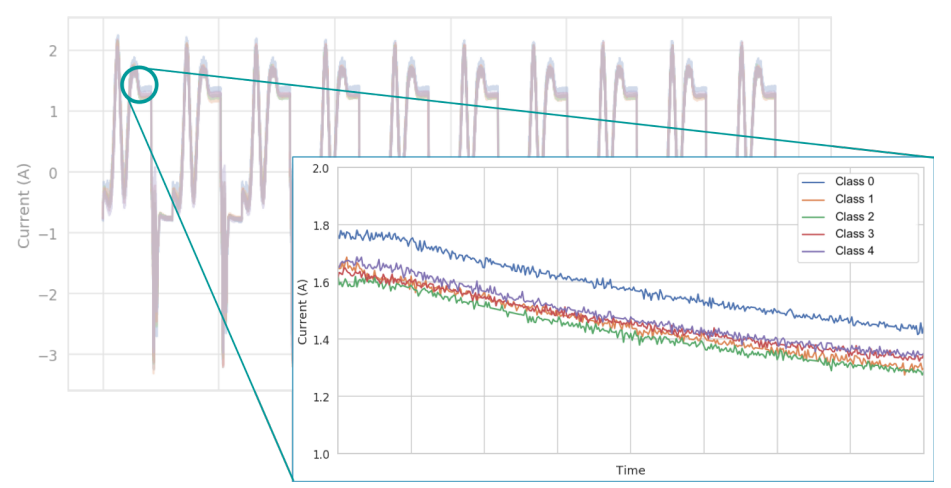

Figure 4. Samples of electricity consumption for different tensioning values.

Table 1. Tension level dataset.

\begin{tabular}{ccc}
\hline Classes & \# of Cycles & Dataset \% \\
\hline 0 & 5952 & $35.30 \%$ \\
\hline 1 & 1591 & $9.44 \%$ \\
\hline 2 & 1937 & $11.70 \%$ \\
\hline 3 & 3174 & $18.82 \%$ \\
\hline 4 & 4172 & $24.74 \%$ \\
\hline Total cycles & 16,862 & $100 \%$ \\
\hline
\end{tabular}

From the experimental results, the analytic service presented in Section 4.1 turns out to be effective in predicting the class relative to the belt tensioning values given measurements of the current amplitude.

Features computation. Each incoming motor current signal has been divided into 24 segments (Figure 5 shows the segments over a sample signal collected from the RobotBox use case), and each segment has been characterized by statistical features (e.g. mean, std, quartiles, Kurtosis, skewness), for a total of 350 features.

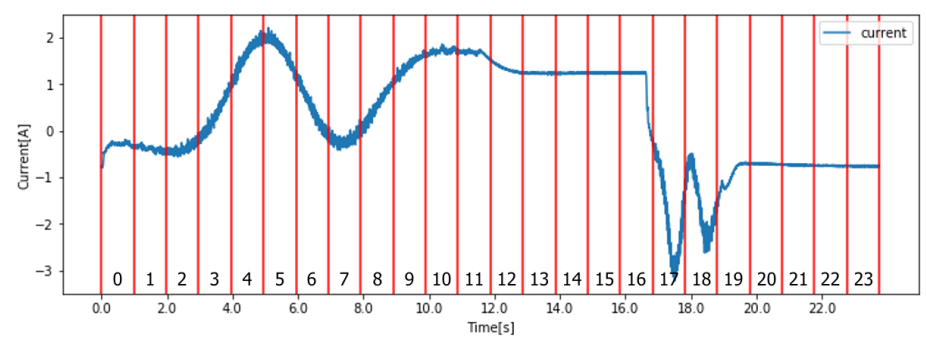

Figure 5. Sample current signal collected from the RobotBox with the segments highlighted.

Features selection. Then, through the correlation test, the number of features is reduced choosing a proper threshold. From this step both the performance and quality of the predictive model will be affected. Figure 6 shows the f-measure obtained without the feature selection step and with two 
different thresholds for the correlation test, 0.3 and 0.5. From the image it is clear that the threshold of 0.5 is outperforming the other two options with all the tested classifier while demonstrating the effectiveness of the proposed feature selection strategy.

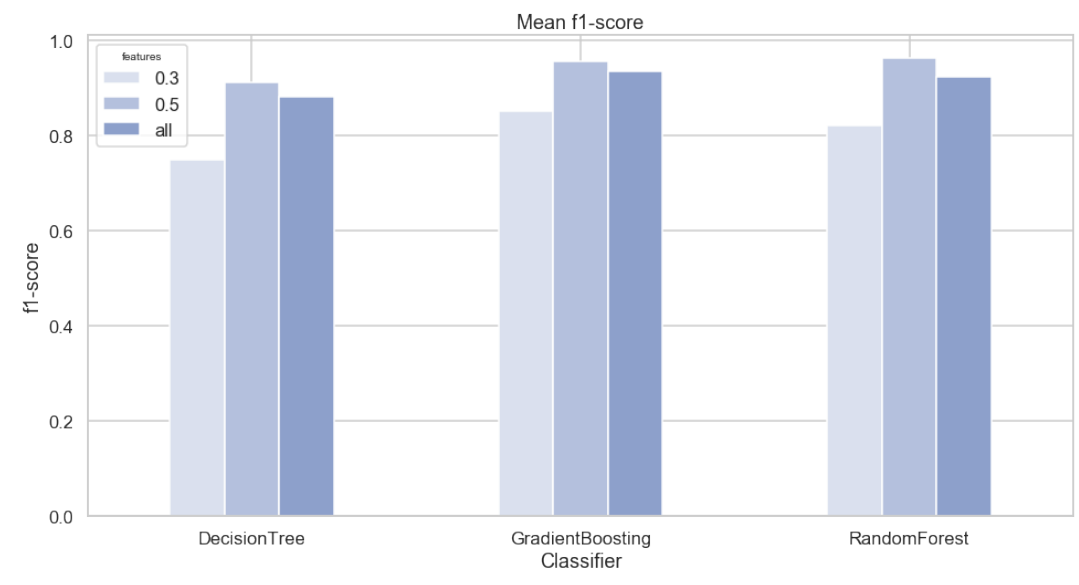

Figure 6. Average f-measure for the different classifiers.

Predictive analytics. The service performs a grid search over three different algorithms available in the MLLib library: Decision Tree (DT), Gradient Boosted Tree (GBT), Random Forest (RF). Each tested configuration has been validated performing a 3 fold cross-validation.

Table 2 shows the performance for each class of the tested classifiers after the grid-search with the stratified k-fold cross-validation.

The grid-search has been performed to analyze an exhaustive range of algorithm configurations including the max-depth of the trees ranging in $\{10,25,50,100,250,500\}$ and the number of estimators ranging in $\{10,25,50,100,250,500\}$ (just in case of RF). Based on the grid-search results, the proposed approach automatically selects the Random Forest (RF) configured with max_depth:10 and n_estimators:250 showing better results for all the classes in term of precision, recall and f-measure. RF and GBT have similar performances despite class 4 where RF is performing slightly better. Table 3 shows the top 10 important features for the RF model along with their feature importance values calculated accordingly to [35]. Feature names are composed by the name of the statistics and the segment identifier on which they have been calculated with the following format: <name_of_the_statistics $>-<$ segment_id $>$. From the feature importance it is possible to notice that the most frequent statistical feature exploited by the RF model is the mean of the absolute change (i.e., the mean of the numerical differences between each couple of consecutive values in the segment) calculated in the first part of the input signals (segments 1, 4, 5, 6 and 10 in Figure 5). Also segment 1 (i.e., at the beginning of the cycle in the acceleration phase) appears three times and segment 10 (i.e., the brake phase before idle phase) two times in the top ten features meaning that these segments are relevant for the prediction process.

The best model is then saved on HDFS or deployed on the edge enabling the real-time prediction step to be available both on the cloud or on premises.

In case of high performance constraints of the production environment, the model, trained on the cloud, can be deployed directly to the edge gateway. In this case the latency of the predictive analytics can be drastically reduced.

In the actual real case scenario, the edge gateway maintains a queue of incoming current signals and runs the edge prediction service that performs both the features computation and the prediction for each new incoming signal. The current implementation of the prediction service on the edge is able to perform the above two tasks (i.e., feature computation and real-time prediction) for multiple incoming signals, making the tasks parallel through a local deployment of the same spark service available in the cloud. However, to test the latency of the predictive analytic service deployed on 
the edge a dual core gateway with 4 GB of ram has been exploited receiving a flow of 1000 RobotBox signals in sequence. To assure its performance a sequential scenario has been simulated in which signals are evaluated in sequence one by one with no delay between each other. The edge prediction service reached, in the sequential scenario, an average computation time of $2.73 \mathrm{~s}$ with a standard deviation of 0.36 . Considering that a robot cycle in the RobotBox use case last around $24 \mathrm{~s}$, the performance reached by the edge prediction service are more than sufficient to have a real-time answer.

Table 2. Classification results, correlation threshold 0.5 .

\begin{tabular}{ccccc}
\hline Classifier & Class & Precision & Recall & F-Measure \\
\hline RF & 0 & 0.99 & 0.99 & 0.99 \\
\hline RF & 1 & 0.99 & 0.99 & 0.99 \\
\hline RF & 2 & 0.99 & 0.99 & 0.99 \\
\hline RF & 3 & 0.98 & 0.96 & 0.97 \\
\hline RF & 4 & 0.84 & 0.93 & 0.85 \\
\hline GBT & 0 & 0.99 & 0.99 & 0.99 \\
\hline GBT & 1 & 0.99 & 0.99 & 0.99 \\
\hline GBT & 2 & 0.99 & 0.99 & 0.99 \\
\hline GBT & 3 & 0.98 & 0.96 & 0.97 \\
\hline GBT & 4 & 0.83 & 0.89 & 0.82 \\
\hline DT & 0 & 0.99 & 0.99 & 0.99 \\
\hline DT & 1 & 0.98 & 0.96 & 0.97 \\
\hline DT & 2 & 0.99 & 0.95 & 0.97 \\
\hline DT & 3 & 0.97 & 0.86 & 0.90 \\
\hline DT & 4 & 0.70 & 0.92 & 0.72 \\
\hline
\end{tabular}

Table 3. Feature importance for the top 10 features extracted from the best RF model trained by the predictive analytics service.

\begin{tabular}{cc}
\hline Feature Name & Importance \\
\hline skewness-1 & 0.048 \\
\hline mean_abs_change-6 & 0.048 \\
\hline mean_abs_change-1 & 0.045 \\
\hline mean_abs_change-5 & 0.044 \\
\hline mean_abs_change-4 & 0.040 \\
\hline mean_abs_change-10 & 0.038 \\
\hline kurtosis-3 & 0.030 \\
\hline mean_abs_change-7 & 0.029 \\
\hline kurtosis-1 & 0.023 \\
\hline third_quartile-10 & 0.023 \\
\hline
\end{tabular}

Self-assessment. The self-assessment service provides information about the reliability of the model, built on the historical data, over time.

To better evaluate the proposed approach, a model considering only the classes of data that usually are known by the domain experts has been generated. This is a likely assumption from the moment that usually, and especially in industrial use cases, for a given machinery, only some behaviors are known. Indeed, monitoring the malfunctioning of a machinery is a difficult task, since having a complete 
description of all the possible wrong behaviors is very complex: this motivation supports the role of this service in the SERENA cloud.

To assess the effectiveness of this service a model has been trained on the tension level dataset exploiting only the elements of classes 2 and 4 since they are supposed to be the known classes at training time. Then, a flow of new incoming data has been simulated exploiting the remaining classes in the dataset i.e., classes 0,1 and 3. The test dataset has been injected in the SERENA platform and the self-assessment service has been triggered at specific timestamps. The test trigger's timestamps has been measured in number of incoming samples to keep the analysis time independent and process independent.

For this reason, the self-assessment service has been triggered following the pattern in Table 4 . The cardinality of the data used to train the predictive model is reported in column Training $\left(t_{0}\right)$, then, for each timestamp $\left(t_{n}\right)$ the number of record for each class label injected is reported. Row New data \% shows the percentage of data that is new w.r.t. the data used to train the model, while row Drift \% reports the percentage of drift included at each timestamp.

Table 4. Self-assessment service, data injection pattern.

\begin{tabular}{cccccccccccc}
\hline Label & Training $\left(\boldsymbol{t}_{\mathbf{0}}\right)$ & $\boldsymbol{t}_{\mathbf{1}}$ & $\boldsymbol{t}_{\mathbf{2}}$ & $\boldsymbol{t}_{\mathbf{3}}$ & $\boldsymbol{t}_{\mathbf{4}}$ & $\boldsymbol{t}_{\mathbf{5}}$ & $\boldsymbol{t}_{\mathbf{6}}$ & $\boldsymbol{t}_{\mathbf{7}}$ & $\boldsymbol{t}_{\mathbf{8}}$ & $\boldsymbol{t}_{\mathbf{9}}$ & $\boldsymbol{t}_{\mathbf{1 0}}$ \\
\hline 0 & - & - & - & - & - & - & 2000 & 4000 & 5952 & 5952 & 5952 \\
\hline 1 & - & - & - & - & - & - & - & - & 48 & 1591 & 1591 \\
\hline 2 & 1500 & 250 & 473 & 473 & 473 & 473 & 473 & 473 & 473 & 473 & 473 \\
\hline 3 & - & - & - & - & - & - & - & - & - & 457 & 3174 \\
\hline 4 & 3000 & - & 400 & 800 & 1172 & 1172 & 1172 & 1172 & 1172 & 1172 & 1172 \\
\hline Tot & 4500 & 250 & 873 & 1273 & 1645 & 1645 & 3645 & 5645 & 7645 & 9645 & 12,362 \\
\hline & New data $\%$ & 5.26 & 16.25 & 22.05 & 26.77 & 26.77 & 44.75 & 55.64 & 62.95 & 68.19 & 73.31 \\
\hline & Drift $\%$ & 0.00 & 0.00 & 0.00 & 0.00 & 0.00 & 24.55 & 39.43 & 49.40 & 56.56 & 63.56 \\
\hline
\end{tabular}

From Table 4 it is possible to notice that from time $t_{1}$ to time $t_{5}$ only data from known classes have been injected, then, starting from time $t_{6}$, class 0 is injected, followed by class 1 (from time $t_{8}$ ) and then by class 3 (from time $t_{9}$ ).

The graphs in Figure 7 are extracted from the SERENA dashboard. They show the degradation of the model trained on classes 2 and 4 over time. Figure $7 \mathrm{a}, \mathrm{b}$ show the percentage of degradation measured by the self-assessment cloud service. From the charts it is possible to notice that until time $t_{5}$ no degradation occurs, reflecting what expected from the injection pattern: only data with a known distribution has been collected by the system and the performance of the model remains unchanged.

Then, at time $t_{6}$ class 0 starts to be recorded by the system, however, this data has an unknown distribution to the model under analysis, since class 0 was not present in the training dataset. At time $t_{6}$ the self-assessment service shows a clear degradation of model performance, correctly identifying the presence of the new unknown distribution of data. The degradation then continues to increase until time $t_{10}$ correctly identifying the increasing of the drift with the new incoming data.

In particular it is possible to notice from Figure 7a that label 2 is the class mostly affected by the presence of concept drift, while label 4 is slightly affected only when new data belonging to the unknown labels 1 and 3 are injected in the system.

Thus, the self-assessment service allows to correctly detect per-class predictive model performance degradation over time.

The interactive dashboard allows also to select a specific timestamp showing the details of the degradation w.r.t. the information learned at training time. It is possible to analyze the details of the silhouette curves calculated by the system at training time (silhouette base) and calculated after 
the arrival of new incoming data (silhouette degraded) comparing their trends in Figure 7c,d for what concern degradation at time $t_{1}$ and Figure $7 \mathrm{e}, \mathrm{f}$ for time $t_{10}$.

Comparing the curves in Figure $7 \mathrm{c}, \mathrm{d}$ they show that no degradation has occurred at time $t_{1}$ since the curves are almost overlapped. While, analyzing the curves in Figure $7 \mathrm{e}, \mathrm{f}$ is possible to notice that the most degraded class is 2 , showing a highly degraded silhouette curve (dotted curve) w.r.t. the base silhouette calculated at training time (continuous curve). Class 4 is instead softly degraded since it was affected mainly by the new classes 1 and 3 .

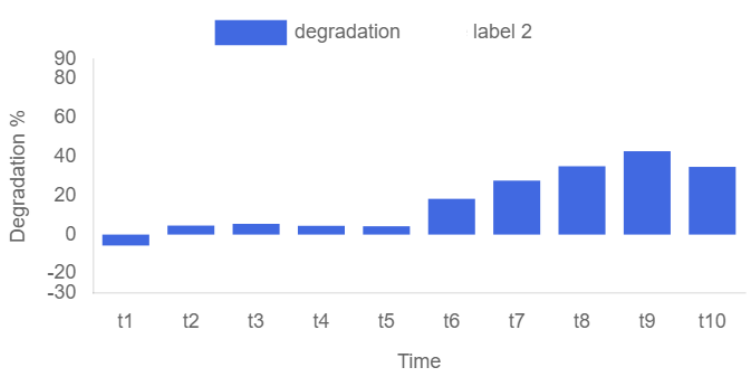

(a) Degradation for class 2 over time.

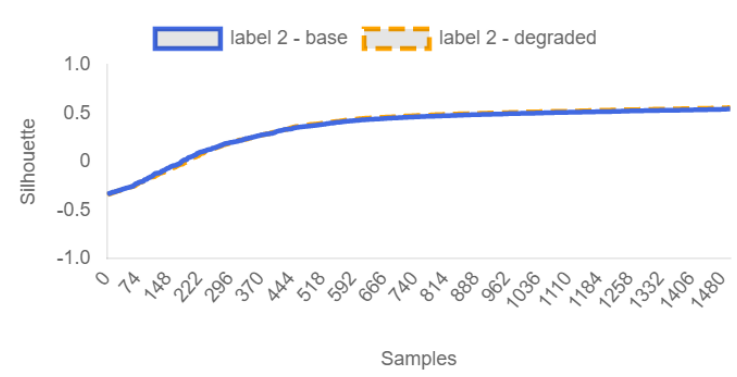

(c) Comparisons of the silhouette curves base, computed at training time, and degraded, computed at time $\mathrm{t} 1$ for label 2.

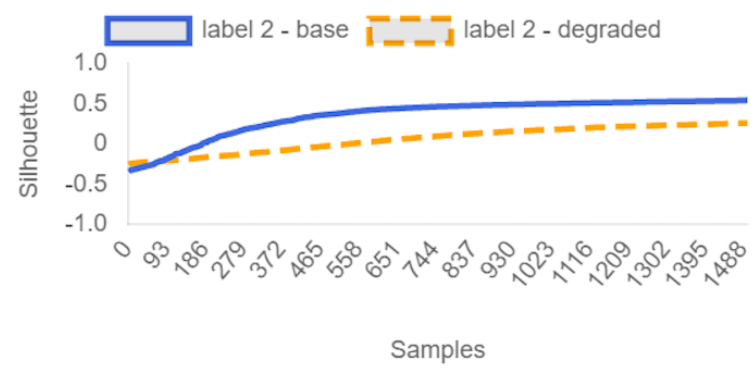

(e) Comparisons of the silhouette curves base, computed at training time, and degraded, computed at time $\mathrm{t} 10$ for label 2.

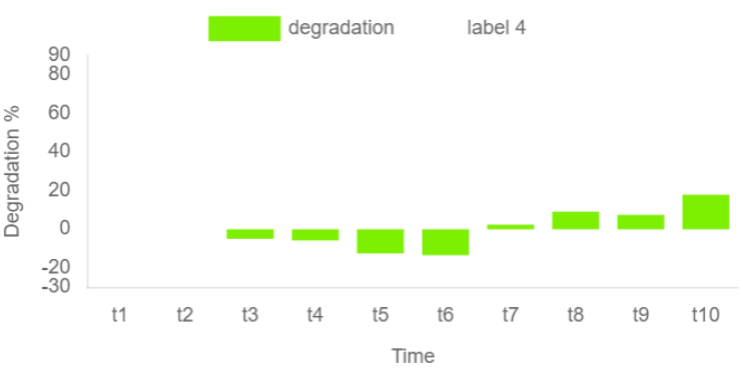

(b) Degradation for class 4 over time.

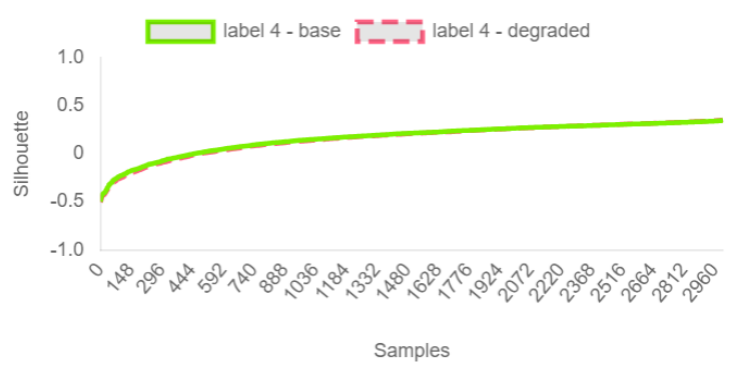

(d) Comparisons of the silhouette curves base, computed at training time, and degraded, computed at time $\mathrm{t} 1$ for label 4 .

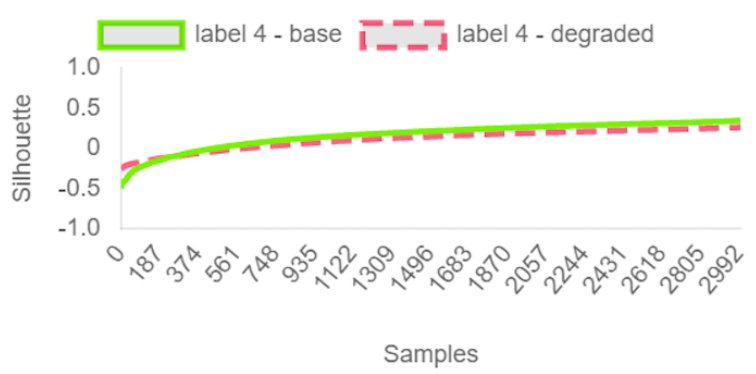

(f) Comparisons of the silhouette curves base, computed at training time, and degraded, computed at time $\mathrm{t} 1$ for label 4.

Figure 7. SERENA cloud dashboard for the self-assessment service.

In this case, a quantitative comparison of the proposed approach with state-of-the-art methodologies proposed to estimate model degradation over time was not performed, since to the best of the authors knowledge none of the available data analytics solutions in literature [36-38] are capable of self-evaluating a predictive model degradation over time when ground-truth class labels are not available. Thus, any comparison to such approaches would not be objective. Techniques like [36] are limited to detect abrupt concept drift due to context changes and they are not able to correctly deal 
with slowly drift as in the context of degrading flows of production data. In addition, strategies like [38] have been developed and tested in very domain specific use cases, while the generality of the approach proposed here has been proved in [29,39]. Furthermore, the proposed concept drift detection strategy has been proved to reach near real-time performance even in big-data environments [39] and to the best of authors knowledge no other solutions addressed this scenario.

Remaining Useful Life (RUL) estimation. The current implementation of the RUL estimation features a tunable approach for estimating the level of degradation of a machinery. In the presented experiments, relevant features to be included in the estimation of the RUL have been defined by a domain expert and validated under a robotic industry use case.

As described in Section 4.1 the motor current signals are split in 24 segments to characterize each phase of the robotic operation. A sample current signal along with its segments is depicted in Figure 5 . When dealing with mechanical machinery, domain experts usually posses the knowledge to recognise the most arduous phases of robot activity that, in the long term, are the cause of its degradation.

In this specific use case the phases in which the motor is breaking down before the idle phase (segments 10 and 11 in Figure 5) have been selected as the phases affecting most its remaining useful life. To summarize the current signals in segments 10 and 11, the means of the current values have been calculated separately for each segment and each RobotBox cycle. Figure 8a,b show respectively the Gaussian kernel densities as estimated for the nominal/correct classes 2 and 4 and for the classes describing the machinery degradation i.e., class 0 . However, in a real-life setting, only the correct functioning of the machinery is known as used in the proposed RUL estimation (i.e., the distribution of classes 2 and 4) while the distribution of signals when the machinery is degrading are not available (i.e., class 0). Nevertheless all of them are reported only to demonstrate that the Gaussian kernel densities represent a simple and easy to understand and interpret approach for summarizing the nominal profile and behavior of an asset versus profiles representing different evolving degradation stages. Given the distribution of the correct and known operational profiles (i.e. classes 2 and 4) the probability of a new robot cycle, in terms of current mean value in segments 10 and 11 , to belong to the distribution of classes 2 and 4 is an indication of whether the robot cycle under analysis is still included in a correct range of values or not and its probability. In other words, if the probability of a feature (current mean in segment 10) belonging to the distribution of classes 2 or 4 is high it can be interpreted that the RobotBox is still working as expected. Then if the probability decreases over time it means that the RobotBox is gradually moving to higher degradation levels, thus the probability distribution of the current signals probably fits under class 0 .

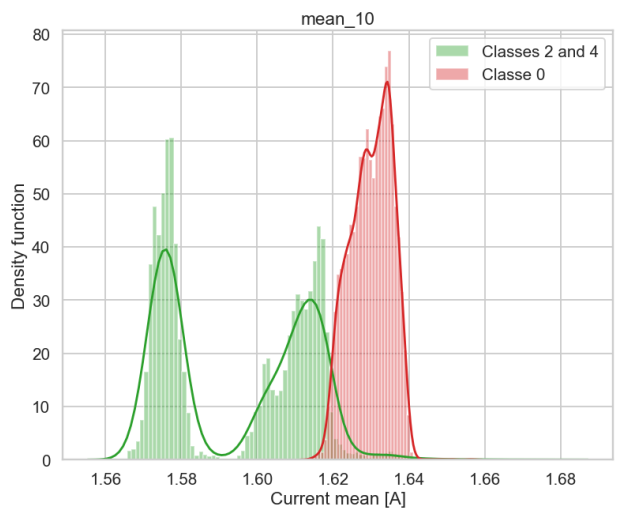

(a) Distribution of the mean of the current at segment 10 .

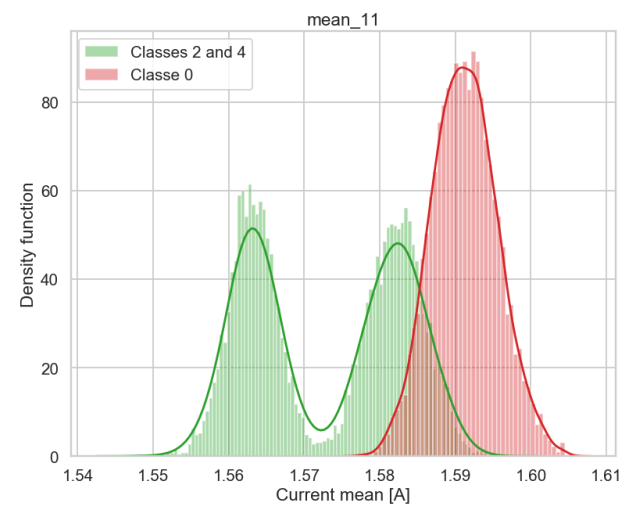

(b) Distribution of the mean of the current at segment 11 .

Figure 8. Distributions of the mean of the current signals for segments 10 and 11 for classes 2 and 4 describing the correct functioning of the machinery and for class 0 describing the incorrect functioning of the machinery. The density has been computed exploiting the Gaussian kernel density estimation. 
To simulate a real scenario for the estimation of the RUL of the RobotBox, where only its correct functioning is known, the same data injection pattern described in Table 4 has been exploited from time $t_{1}$ to time $t_{7}$ using classes 2 and 4 to simulate the correct functioning of the machine and class 0 to simulate the its incorrect functioning.

In particular, in the context of SERENA, the Gaussian distribution of the current mean value in segments 10 and 11 is calculated separately by analyzing all historical signals collected from the beginning of the RobotBox life to time $t_{0}$. Then, for each timestamp $t_{n}$ the percentage of RUL is estimated via Equation (2) with new data from each cycle as shown in Table 4.

Figure 9 shows the RUL value estimated at each timestamp from $t_{1}$ to $t_{7}$ along with their error bands. As expected, the trend of the remaining useful life is clearly descending over time. At the time $t_{1}$ only data belonging to the correct functioning is collected showing an estimated RUL of almost $62 \%$ with an error ranging between $40 \%$ and $85 \%$. As soon as more data belonging to the correct distribution are collected the RUL slowly decreases as a result of the RobotBox's natural degradation. Then, starting from time $t_{6}$, data belonging to the wrong (unknown) functioning of the machine are injected and the RUL value is decreased to $13.30 \%$ at time $t_{6}$ and to $7.18 \%$ at time $t_{7}$. These results were assessed with domain experts in the robotics industry, verifying that the high error in the RUL estimation in time $t_{1}$ is a result of the limited number of robot cycles analyzed.

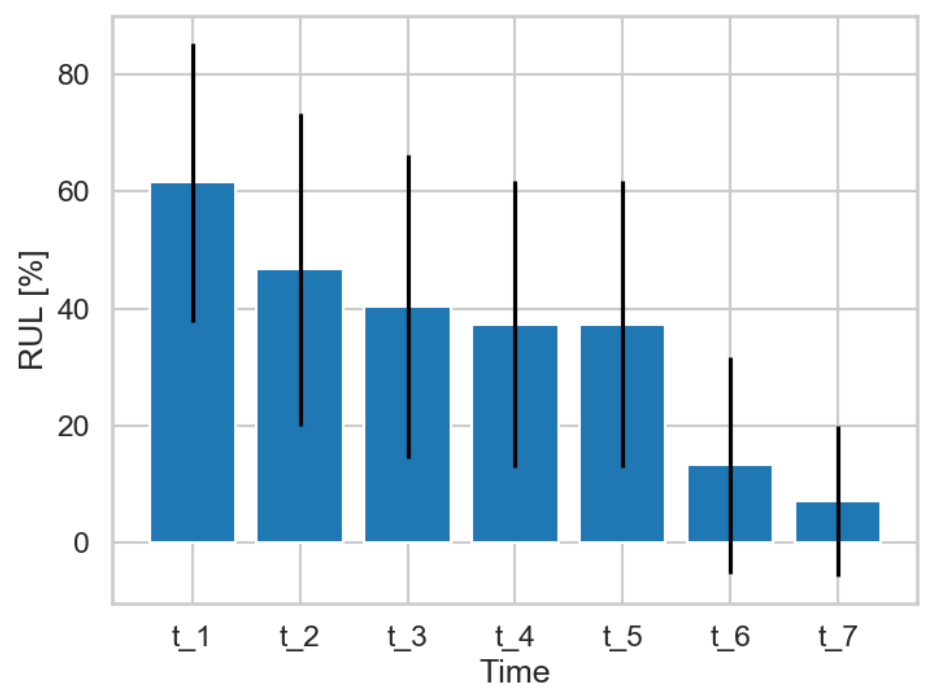

Figure 9. Remaining Useful Time percentage estimated by SERENA over time. The data injection pattern refers to the one showed in Table 4 from time $t_{1}$ to time $t_{7}$.

Scheduling. In the current experiment, a schedule triggered by a RUL estimation, as provided by the predictive analytics service, was generated in approximately $11 \mathrm{msec}$, and included the execution of two tasks; machine inspection and replacement of the gearbox, along with three potential resources; (1) a team of one newcomer and one of middle experience, (2) one newcomer and one expert and (3) one expert. The difference in task completion time as well as their cost are presented in the Table 5, per task. 
Table 5. Information used by the scheduling service for the experiment.

\begin{tabular}{cccc}
\hline Operator & Task & Minutes & Euros/Minute \\
\hline Newcomer, Middle & Machine inspection & 20 & 0.25 \\
\hline Newcomer, Expert & Machine inspection & 120 & 0.25 \\
\hline Expert & Machine inspection & 15 & 0.4 \\
\hline Newcomer, Middle & Replace the gearbox & 100 & 0.4 \\
\hline Newcomer, Expert & Replace the gearbox & 10 & 0.5 \\
\hline Expert & Replace the gearbox & 80 & 0.5 \\
\hline
\end{tabular}

3D model visualization. This service is part of the SERENA cloud, and visualizes the 3D model of the RobotBox, along with its real time movement, prediction information and a 3D virtual maintenance procedure. Figure 10 shows a screenshot of the service interface that includes the real time position chart of the axis of rotation, which at runtime is mirrored by the movement of the yellow arm. The top bar provides links to: the "Charts" panel, which presents visual charts of the analytics information coming from the prediction service; and the "Maintenance" panel, which provides a step-by-step animated guide.

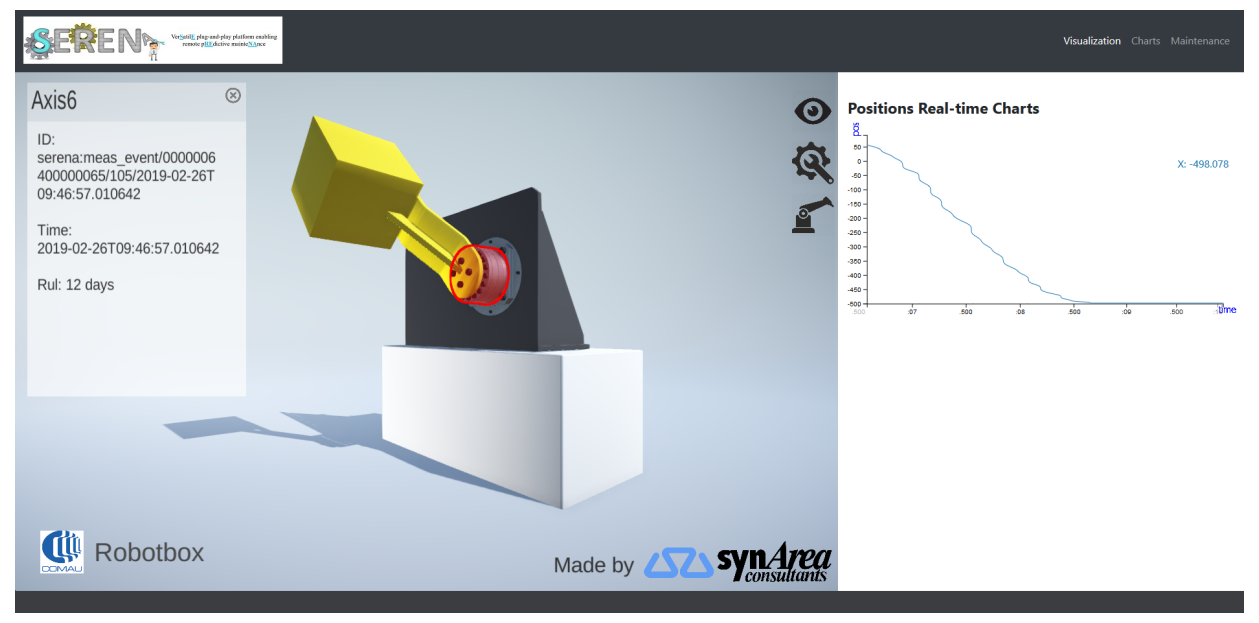

Figure 10. Visualization service screenshot.

\section{Conclusions and Future Applications}

This paper presents a lightweight architecture merging cloud based and edge deployed components towards a first end-to-end implementation of a predictive analytics platform, with respect to cyber-physical features and the vision of Industry 4.0. In this regard, the proposed architecture has been designed with respect to some well-established needs of industrial enterprises, such as compatibility with both the on-premise and the in-the-cloud environments, exploitation of reliable and largely supported big data platforms, high levels of horizontal scalability, easy deployment through containerized software modules.

In addition, a novel and generic methodology for RUL estimation towards enabling predictive maintenance activities in a cyber physical production system is introduced. Different operational profiles of the monitored asset(s) are created and compared to a nominal one, created at the very beginning of the assets setup and operation. It should be noted that the proposed approach supports the connection of the data collected for the RUL estimation to the existing maintenance plan, optimising the amount of data collected. For example, considering a conventional maintenance plan of two weeks, positive results out of the proposed approach can be demonstrated with a dataset of only two weeks. in this way, the proposed approach is capable of being applied to a versatile set of domains and assets, without requiring extensive and costly monitoring systems and numerous sensors. 
A prototype has been implemented and validated in an industrial use case concerning the predictive maintenance of a robotic manipulator. A set of services has been integrated to evaluate the proposed architecture and its potential. The results demonstrate that the integrated solution was able to bridge the gap between machine data acquisition and cloud processing and enable the generation of predictive analytics and strategies. The use of containerization technologies poses additional effort for industrial applications, including workload balancing, automating deployments, auto-discovery of nodes, as well as the configuration effort for creating the cluster. As the proposed approach is not constrained to any specific set of technologies, it has the ability to evolve as the project progresses into the next phase.

In conclusion, future work will focus on integrating additional functionalities to the overall architecture, such as data security features and increasing the robustness of the integrated solution. As it regards the data analytics, further investigation and research is required to effectively analyze different kinds of industrial processes (including those that are very slowly degrading over time) and being able to perform the prediction with different time horizons. Moreover, since the analytics part is a critical aspect for enabling predictive maintenance solutions, particular focus will be given on testing and improving the proposed approaches in the context of other real-life settings, on both on-premise and (public) cloud.

Author Contributions: Data curation, L.M. and C.N.; Methodology, N.N., T.C. and S.P. (Simone Panicucci); Software, D.B., F.V., S.P. (Stefano Proto), P.B., G.C. and S.A.; Validation, S.P. (Simone Panicucci), F.V. and S.P. (Stefano Proto); Writing-original draft, N.N. and T.C.; Writing-review \& editing E.M., S.M., N.O. and A.M. All authors have read and agreed to the published version of the manuscript.

Funding: The research leading to these results has been partially funded by European Commission under the H2020-IND-CE-2016-17 program, FOF-09-2017, Grant agreement No. 767561 "SERENA" project, VerSatilE plug-and-play platform enabling REmote predictive mainteNAnce.

Conflicts of Interest: The authors declare no conflict of interest.

\section{References}

1. Apiletti, D.; Barberis, C.; Cerquitelli, T.; Macii, A.; Macii, E.; Poncino, M.; Ventura, F. iSTEP, an Integrated Self-Tuning Engine for Predictive Maintenance in Industry 4.0. In Proceedings of the IEEE International Conference on Parallel \& Distributed Processing with Applications, Ubiquitous Computing \& Communications, Big Data \& Cloud Computing, Social Computing \& Networking, Sustainable Computing \& Communications, ISPA/IUCC/BDCloud/SocialCom/SustainCom 2018, Melbourne, Australia, 11-13 December 2018; pp. 924-931.

2. Niño, M.; Blanco, J.M.; Illarramendi, A. Business understanding, challenges and issues of Big Data Analytics for the servitization of a capital equipment manufacturer. In Proceedings of the IEEE International Conference on Big Data (Big Data), Santa Clara, CA, USA, 29 October-1 November 2015; pp. 1368-1377.

3. Marques, G.; Pitarma, R.M.; Garcia, N.; Pombo, N. Internet of Things Architectures, Technologies, Applications, Challenges, and Future Directions for Enhanced Living Environments and Healthcare Systems: A Review. Electronics 2019, 8, 1081. [CrossRef]

4. Miškuf, M.; Zolotová, I. Comparison between multi-class classifiers and deep learning with focus on industry 4.0. In Proceedings of the Cybernetics Informatics (K I), Levoca, Slovakia, 2-5 February 2016; pp. 1-5.

5. Apiletti, D.; Baralis, E.; Cerquitelli, T.; Garza, P.; Pulvirenti, F.; Venturini, L. Frequent itemsets mining for Big Data: A comparative analysis. Big Data Res. 2017, 9, 67-83. [CrossRef]

6. Babiceanu, R.F.; Seker, R. Big Data and virtualization for manufacturing cyber-physical systems: A survey of the current status and future outlook. Comput. Ind. 2016, 81, 128-137. [CrossRef]

7. Huang, Z.; Zhong, A.; Li, G. On-Demand Processing for Remote Sensing Big Data Analysis. In Proceedings of the IEEE International Symposium on Parallel and Distributed Processing with Applications and 2017 IEEE International Conference on Ubiquitous Computing and Communications (ISPA/IUCC), Guangzhou, China, 12-15 December 2017; pp. 1241-1245. 
8. Simonis, I. Container-based architecture to optimize the integration of microservices into cloud-based data-intensive application scenarios. In Proceedings of the 12th European Conference on Software Architecture: Companion Proceedings, Madrid, Spain, 24-28 September 2018; ACM: New York, NY, USA, 2018; p. 34.

9. Acquaviva, A.; Apiletti, D.; Attanasio, A.; Baralis, E.; Bottaccioli, L.; Cerquitelli, T.; Chiusano, S.; Macii, E.; Patti, E. Forecasting Heating Consumption in Buildings: A Scalable Full-Stack Distributed Engine. Electronics 2019, 8, 491. [CrossRef]

10. Jin, Y.; Lee, H. On-Demand Computation Offloading Architecture in Fog Networks. Electronics 2019, 8, 1076. [CrossRef]

11. Xiang, Y.; Zhu, Z.; Coit, D.W.; Feng, Q. Condition-based Maintenance Under Performance-based Contracting. Comput. Ind. Eng. 2017, 111, 391-402. [CrossRef]

12. Mehta, P.; Werner, A.; Mears, L. Condition Based Maintenance-systems Integration and Intelligence Using Bayesian Classification and Sensor Fusion. J. Intell. Manuf. 2015, 26, 331-346. [CrossRef]

13. Murphree, J. Machine learning anomaly detection in large systems. In Proceedings of the IEEE AUTOTESTCON, Anaheim, CA, USA, 12-15 September 2016; pp. 1-9.

14. Zhang, C.; Lim, P.; Qin, A.K.; Tan, K.C. Multiobjective Deep Belief Networks Ensemble for Remaining Useful Life Estimation in Prognostics. IEEE Trans. Neural Netw. Learn. Syst. 2016, 1-13. [CrossRef] [PubMed]

15. Asmai, S.A.; Basari, A.S.H.; Shibghatullah, A.S.; Ibrahim, N.K.; Hussin, B. Neural network prognostics model for industrial equipment maintenance. In Proceedings of the 11th International Conference on Hybrid Intelligent Systems (HIS), Melacca, Malaysia, 5-8 December 2011; pp. 635-640.

16. Chen, Y.; Zhu, F.; Lee, J. Data quality evaluation and improvement for prognostic modeling using visual assessment based data partitioning method. Comput. Ind. 2013, 64, 214 - 225. [CrossRef]

17. Bowden, D.; Marguglio, A.; Morabito, L.; Napione, C.; Panicucci, S.; Nikolakis, N.; Makris, S.; Coppo, G.; Andolina, S.; Macii, A.; et al. A Cloud-to-edge Architecture for Predictive Analytics. In Proceedings of the Workshops of the EDBT/ICDT 2019 Joint Conference, EDBT/ICDT 2019, Lisbon, Portugal, 26 March 2019.

18. JSON-LD. Available online: https://json-ld.org/ (accessed on 15 November 2019).

19. MIMOSA. Available online: http://www.mimosa.org/specifications/osa-eai-3-2-3/ (accessed on 15 November 2019).

20. Shvachko, K.; Kuang, H.; Radia, S.; Chansler, R. The Hadoop Distributed File System. In Proceedings of the 2010 IEEE 26th Symposium on Mass Storage Systems and Technologies (MSST), Ilan, Taiwan, 18-20 September 2010; IEEE Computer Society: Washington, DC, USA, 2010; pp. 1-10.

21. Lakshman, A.; Malik, P. Cassandra: A Decentralized Structured Storage System. SIGOPS Oper. Syst. Rev. 2010, 44, 35-40. [CrossRef]

22. Proto, S.; Ventura, F.; Apiletti, D.; Cerquitelli, T.; Baralis, E.; Macii, E.; Macii, A. PREMISES, a Scalable Data-Driven Service to Predict Alarms in Slowly-Degrading Multi-Cycle Industrial Processes. In Proceedings of the IEEE International Congress on Big Data, BigData Congress, Milan, Italy, 8-13 July 2019; pp. 139-143.

23. Ross, S.M. Introduction to Probability Models; Academic Press: Cambridge, MA, USA, 2014.

24. Zaharia, M.; Xin, R.S.; Wendell, P.; Das, T.; Armbrust, M.; Dave, A.; Meng, X.; Rosen, J.; Venkataraman, S.; Franklin, M.J.; et al. Apache Spark: A Unified Engine for Big Data Processing. Commun. ACM 2016, 59, 56-65. [CrossRef]

25. Meng, X.; Bradley, J.; Yavuz, B.; Sparks, E.; Venkataraman, S.; Liu, D.; Freeman, J.; Tsai, D.; Amde, M.; Owen, S.; et al. MLlib: Machine Learning in Apache Spark. J. Mach. Learn. Res. 2016, 17, 1235-1241.

26. Breiman, L. Classification and Regression Trees; Routledge: London, UK, 2017.

27. Breiman, L. Random Forests. Mach. Learn. 2001, 45, 5-32.:1010933404324. [CrossRef]

28. Friedman, J.H. Greedy function approximation: A gradient boosting machine. Ann. Stat. 2001, 1189-1232. [CrossRef]

29. Ventura, F.; Proto, S.; Apiletti, D.; Cerquitelli, T.; Panicucci, S.; Baralis, E.; Macii, E.; Macii, A. A New Unsupervised Predictive-Model Self-Assessment Approach That SCALEs. In Proceedings of the IEEE International Congress on Big Data, BigData Congress, Milan, Italy, 8-13 July 2019; pp. 144-148.

30. Kim, S.; Kim, H. A new metric of absolute percentage error for intermittent demand forecasts. Int. J. Forecast. 2016, 32, 669-679. [CrossRef]

31. Kleinbaum, D.G.; Klein, M. Survival Analysis; Springer: Berlin, Germany, 2010; Volume 3. 
32. Parzen, E. On Estimation of a Probability Density Function and Mode. Ann. Math. Statist. 1962, 33, $1065-1076$. [CrossRef]

33. Nikolakis, N.; Papavasileiou, A.; Dimoulas, K.; Bourmpouchakis, K.; Makris, S. On a versatile scheduling concept of maintenance activities for increased availability of production resources. In Proceedings of the 6th CIRP Global Web Conference-Envisaging the Future Manufacturing, Design, Technologies and Systems in Innovation Era (CIRPe 2018), Stockholm, Sweden, 16-18 May 2018; pp. 172-177.

34. Node-RED. Available online: https://nodered.org/ (accessed on 15 November 2015).

35. Hastie, T.; Tibshirani, R.; Friedman, J. The Elements of Statistical Learning: Data Mining, Inference, and Prediction; Springer Science \& Business Media: Berlin, Germany, 2009.

36. Vorburger, P.; Bernstein, A. Entropy-based Concept Shift Detection. In Proceedings of the Sixth International Conference on Data Mining (ICDM'06), Hong Kong, China, 18-22 December 2006; pp. 1113-1118. [CrossRef]

37. Bifet, A.; Gavalda, R. Learning from time-changing data with adaptive windowing. In Proceedings of the 2007 SIAM International Conference on Data Mining, Minneapolis, MN, USA, 26-28 April 2007; pp. 443-448.

38. Klinkenberg, R.; Joachims, T. Detecting Concept Drift with Support Vector Machines; In Proceedings of the International Conference on Machine Learning, Stanford, CA, USA, 29 June-2 July 2000; pp. 487-494.

39. Cerquitelli, T.; Proto, S.; Ventura, F.; Apiletti, D.; Baralis, E. Towards a Real-Time Unsupervised Estimation of Predictive Model Degradation. In Proceedings of the Real-Time Business Intelligence and Analytics, BIRTE 2019, Los Angeles, CA, USA, 26 August 2019; Association for Computing Machinery: New York, NY, USA, 2019. [CrossRef]

(C) 2020 by the authors. Licensee MDPI, Basel, Switzerland. This article is an open access article distributed under the terms and conditions of the Creative Commons Attribution (CC BY) license (http://creativecommons.org/licenses/by/4.0/). 